\title{
Post-embryonic development of remipede crustaceans
}

\author{
Stefan Koenemann, a,* Frederick R. Schram, ${ }^{\mathrm{b}}$ Armin Bloechl, ${ }^{a}$ Thomas M. Iliffe,c Mario Hoenemann, ${ }^{\mathrm{a}}$ \\ and Christoph Heldd \\ ${ }^{a}$ Institute for Animal Ecology and Cell Biology, University of Veterinary Medicine Hannover Foundation, Bünteweg 17d, 30559 \\ Hannover, Germany \\ ${ }^{\mathrm{b}}$ Department of Biology, University of Washington, Seattle, WA 98195, USA \\ ${ }^{c}$ Department of Marine Biology, Texas A\&M University at Galveston, Galveston, TX 77553-1675, USA \\ ${ }^{\mathrm{d}}$ Stiftung Alfred-Wegener-Institut für Polar- und Meeresforschung, Columbusstraße, 27568 Bremerhaven, Germany \\ *Author for correspondence (email: stefan.koenemann@tiho-hannover.de)
}

SUMMARY During diving explorations of anchialine cave systems on Abaco Island, Bahamas, we collected five larvae that represent different developmental stages of remipede crustaceans. Based on four early naupliar stages and a post- naupliar larva, it is possible for the first time to reconstruct the postembryonic development of Remipedia some 25 years after their discovery. These specimens begin to fill in some critical gaps in our knowledge of this important group of crustaceans.

\section{INTRODUCTION}

The discovery and identification of larvae has often been a critical step in crustacean studies toward properly placing otherwise unique groups. The most outstanding example involved the affinities of barnacles. In the early 1800s, the scientific community argued as to whether or not barnacles were molluscs until Thompson (1830) discovered their larvae and described for the first time their metamorphosis into cirripedes and, thus, identified them as Crustacea.

Yager (1981) described the first species of the cave-dwelling Remipedia, and its recognition as a new class of Crustacea reanimated long-standing controversies on arthropod evolution (Schram et al. 1986; McKenzie 1991; Boxshall et al. 1992; Moura and Christoffersen 1996; Emerson and Schram 1997; Wills 1997; Schram and Hof 1998). The remipedes exhibit a number of autapomorphies, that is, unique features not shared with any other group of crustaceans. These include three pairs of large, subchelate postmandibular mouthparts. The hypodermic claw of the maxillule is connected to a large gland that is used in a predatory mode of feeding (Schram and Lewis 1989; van der Ham and Felgenhauer 2007). In addition, the remipede trunk, composed of up to 42 somites (Koenemann et al. 2006), is not subdivided into separate tagmata, for example, into thorax and pleon as in Malacostraca, or thorax and abdomen as in Branchiopoda, Cephalocarida, and Maxillopoda (Schram and Koenemann 2004a).

It has been argued that the long, homonomously segmented trunk region in Remipedia may represent an ancestral ground pattern in the evolution of Crustacea (e.g., Schram
1983). This hypothesis grows out of long-held views of the evolution of arthropods (Snodgrass 1952). On the other hand, several studies suggest that remipedes possibly occupy a more advanced position within the Crustacea (Fanenbruck et al. 2004; Schram and Koenemann 2004b).

The sources for these disagreements arise from the fact that many aspects of the biology of Remipedia remain blank spots on a map. For example, modes of both reproduction and development have been entirely unknown until now. The smallest individuals of various species previously recorded were $3-4 \mathrm{~mm}$ long, with trunks composed of at least 15 somites, whereas general Bauplan characteristics were comparable to those of adults. Now, during diving explorations of anchialine caves on Abaco Island, Bahamas, we have collected larvae that represent earlier developmental stages of Remipedia (Fig. 1). Based on the discovery of five larval stages, we at last can begin to reconstruct the early development of Remipedia.

\section{MATERIALS AND METHODS}

The larvae were collected by divers in two anchialine caves on Abaco Island directly from the water column below the halocline. The orthonauplius, metanauplius 1 and the post-naupliar larva were collected from one cave in depths between 18 and $45 \mathrm{~m}$; metanauplii 2 and 3 came from another cave in 10-41 m depths. All specimens were preserved in $96 \%$ EtOH immediately after collection. One metanauplius (MN-1) was embedded in glycerine gelantine for confocal laser scanning microscopy (CLSM). CLSM images were obtained at two different excitation wavelengths via autofluorescence. The drawing of the postnauplius was produced 

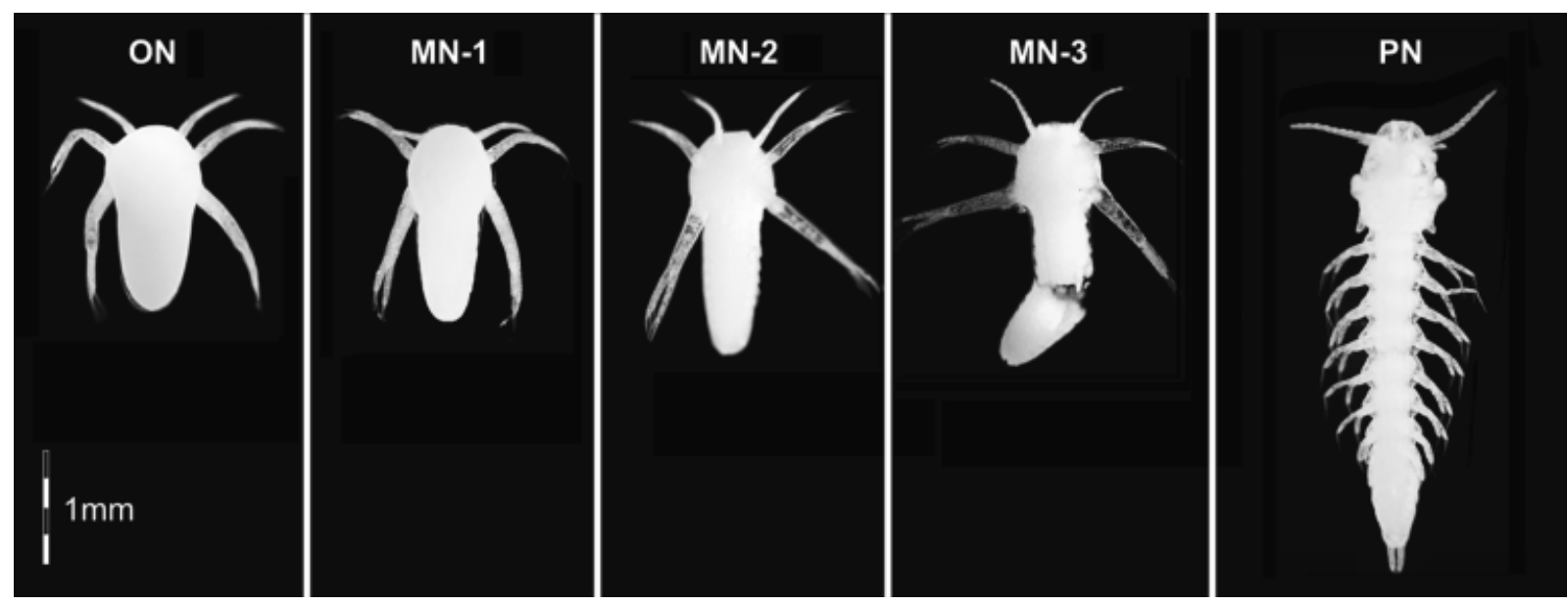

Fig. 1. Early developmental stages of Remipedia. ON, orthonauplius; MN, metanauplius; PN, post-naupliar larva.

using a light microscope with a mirror-reflex tube; the reconstruction drawing of the metanauplius MN-3 was based on photographs and light microscopy.

\section{POST-EMBRYONIC DEVELOPMENT}

We have at hand five free-living larvae that represent different developmental stages: one orthonauplius, three metanauplii, and one postnauplius. All five specimens are nonfeeding, lecithotrophic larvae, that is, they rely on yolk as their main energy resource.

The ortho- and metanaupliar larvae bear three pairs of well-developed head appendages that are not articulated.

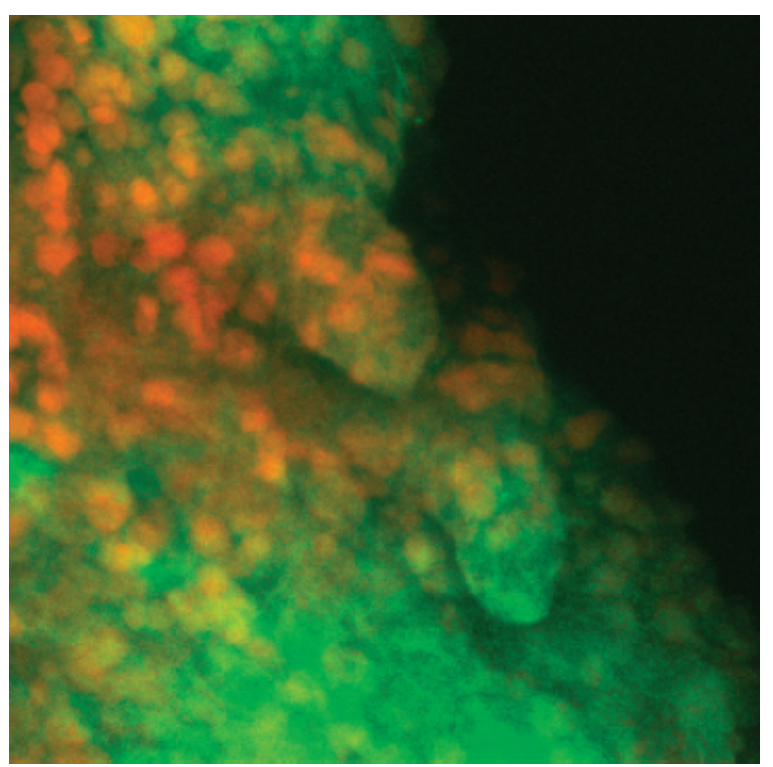

Fig. 2. Detail of posterior head region of metanauplius 1 (ventral view). Anlagen of second maxilla and maxilliped develop as uniramous limb buds. Photo by confocal laser scanning microscopy.
However, the relatively short and thin first antenna is uniramous, whereas the second antenna and the massive mandible have biramous distal palps. The larvae lack cephalic shields and median (naupliar) eyes, and there is no Anlage of a labrum. The stomodaeum and proctodaeum are incipiently developed as small, short invaginations. In the larger metanauplii, the invaginated proctodaeum is more marked, and two knob-like, incipient caudal rami appear on the posterior body terminus.

The orthonauplius larva (ON, Fig. 1) has an inverted pearshaped body (length $1.66 \mathrm{~mm}$, maximum width $0.8 \mathrm{~mm}$ ), with a sphere-shaped head region and an elongated posterior extension of the body. The proximal sections of the first and second antennae are expanded; a faint suture is recognizable between proximal and distal sections. The bifurcations of the second antenna and the long and massive mandible begin at ca. $2 / 3$ of the total lengths. Although additional limbs or segments

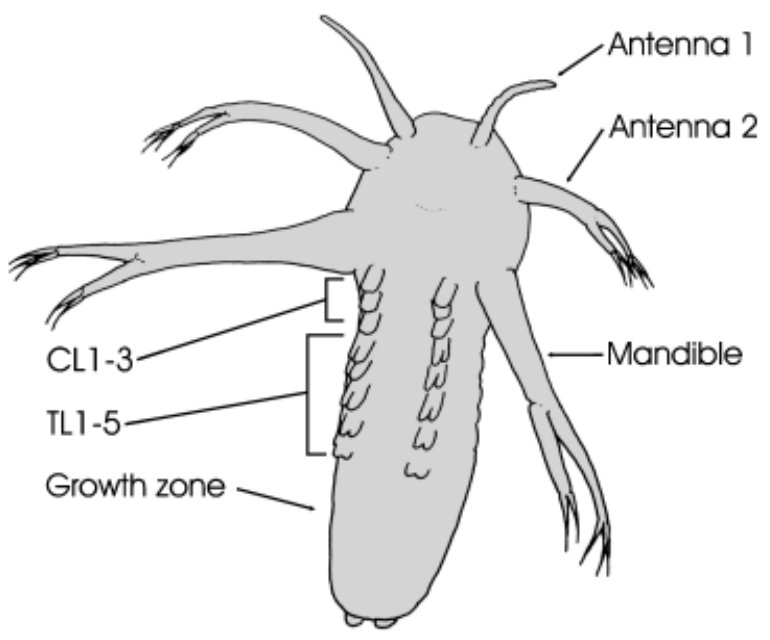

Fig. 3. Drawing of $2.2 \mathrm{~mm}$ metanauplius (MN-3). CL, cephalic limbs (maxilla 1 and 2, maxilliped); TL, trunk limbs. 
are not present, there are five to six paired, dark stripes visible in the ventral body region posterior to the mandibles.

The metanauplius 1 (MN-1, Figs. 1 and 2) has a body length of $1.72 \mathrm{~mm}$ and a maximum width of $0.76 \mathrm{~mm}$. The three pairs of naupliar limbs are similar to those in the orthonauplius. However, the posterior region is longer than in the $\mathrm{ON}$, with four to five somites beginning to differentiate, and a dorsal fold separating head and trunk. There are three pairs of uniramous limbs that lie within the presumptive head region: first and second maxilla, and maxilliped. In addition, there are three pairs of bilobed limb buds along the ventral trunk posterior to the uniramous limb buds.

The metanauplius 2 (MN-2, Fig. 1) has a body length of $1.96 \mathrm{~mm}$, the maximum width is $0.76 \mathrm{~mm}$. The naupliar limbs are comparable to those in $\mathrm{ON}$ and $\mathrm{MN}-1$, but the distal parts of the two branches in second antenna and mandible are further developed. The prospective trunk region bears six pairs of limb Anlagen. Two small buds appear terminally on the developing anal somite.

MN-3 (Figs. 1 and 3) is the largest metanauplius, with a body length of $2.2 \mathrm{~mm}$ and a maximum width of $0.76 \mathrm{~mm}$. Compared with the smaller nauplii, the second antenna and mandible are even further developed. There are eight pairs of biramous limb buds along the ventral trunk region, and the terminal caudal rami have grown larger.

The postnaupliar larva (PN, Figs. 1 and 4) has a body length of $3.75 \mathrm{~mm}$ and a maximum width of $0.6 \mathrm{~mm}$. Based on several distinct characters, including two prominent posterolateral projections of the cephalic shield, this larva can be identified as belonging to a species of the remipede genus Pleomothra. Although the post-oral head limbs are generally less developed than the antennae and the first six pairs of trunk appendages, the metamorphosis of the cephalon from metanauplius to this stage is apparent. Two frontal filaments with small processes have grown in the anterior head region between the first antennae. The first antennae are biramous, each composed of a dorsal flagellum of 10 articles and a short unsegmented ventral flagellum. The second antenna resembles the biramous appendage characteristic of the adult morphology. The most conspicuous head limb is the mandible that still bears a biramous palp, but a bulbous basal endite reveals the forthcoming location of the gnathobase. The labrum appears as an Anlage, and the invagination of the stomatodaeum is still incomplete. The three pairs of prehensile cephalic appendages, first and second maxilla and maxilliped, exhibit incipient articulations, although the characteristic elbows of these limbs are already present; endites and terminal claws are not fully developed. The anterior trunk region bears seven pairs of relatively well-developed limbs, followed by three pairs of incipient appendages and a relatively large undifferentiated growth zone. The anal somite with two caudal rami is separated from the growth zone by a faint suture.

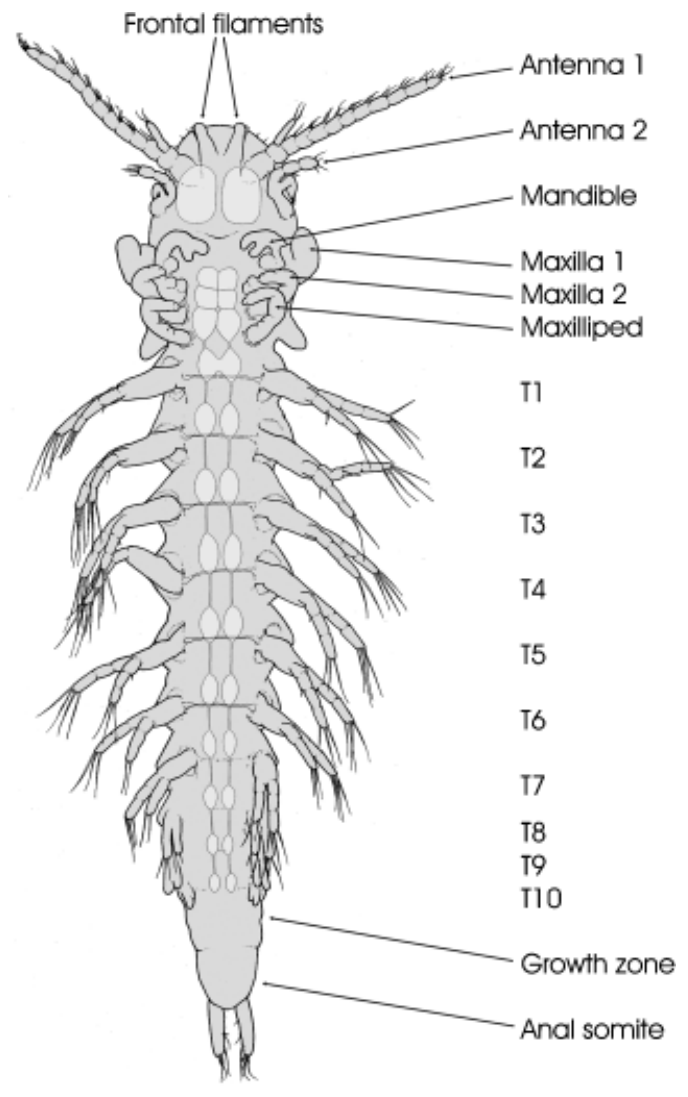

Fig. 4. Drawing of post-naupliar larva (ventral view); T, trunk segment. Please note that ganglia are shown as they appeared in the undissected specimen.

\section{DISCUSSION}

The five larvae now provide for the first time a framework about which to understand remipede development. An orthonauplius followed by a series of metanauplii lead to a postnaupliar stage. Although the development is gradual, it is not stepwise. Rather, each stage involves small quantum additions of segments and limbs. A gap remains between the postnauplius, with 10 segments, and a (feeding) juvenile with 15 trunk segments (Koenemann et al. 2006). We assume that this gap is filled by a series of molts (perhaps as seen in the Anostraca) rather than by a single quantum molt, but only further collection and study will allow a definitive answer.

However, one critical point concerns the nonfeeding, lecithotrophic nature of the free-living remipede larvae. Scholtz (2000) pointed out that the free-living orthonauplii of Euphausiacea and Dendrobranchiata - both decapod Malacostraca-do not feed, that is, that they are lecithotrophic. Most other crustacean orthonauplii generally do feed. Scholtz hypothesized that the free-living eumalacostracan nauplii were not "homologous" to those of branchiopods and maxillopodans, but rather represent derived larval stages in a major 
taxon that have secondarily evolved from egg-nauplii. To support his hypothesis, Scholtz provided a number of characteristics of euphausiacean and dendrobranchiate nauplii that deviate from those of other free living, feeding crustacean naupliar larvae (which are presumed to represent a plesiomorphic feature of the Crustacea s. lat.). These include a yolkrich body; both stomatodaeum and proctodaeum with only short invaginations; the absence of a labrum Anlage, a mandibular gnathobase and a masticatory spine on the second antenna; nonarticulated naupliar appendages; and an undifferentiated pre-anal growth zone.

The lecithotrophic remipede nauplii described herein share all of these characters with euphausiaceans and dendrobranchiates. However, there are a few important characters that remipede larvae do not share with free-living malacostracan nauplii, for example, three pairs of uniramous cephalic limbs followed by biramous trunk limbs and a posterior body terminus with developing caudal rami on an anal somite (instead of a telson Anlage).

The discovery of free-living lecithotrophic larvae of Remipedia leaves us with several theoretical scenarios for the evolution of crustacean nauplii.

(1) If the free-living malacostracan nauplii are secondarily derived from egg-nauplii (sensu Scholtz), the free-living lecithotrophic naupliar stage may represent a synapomorphy of Remipedia and some Malacostraca. This scenario implies that the free-living remipede nauplii also represent a derived condition, most likely also from an egg-nauplius. At present, there is no evidence to support this assumption.

(2) If, on the other hand, the egg-nauplius in malacostracans corresponds with a derived mode of development, and the free-living lecithotrophic nauplius is the ancestral condition, then remipedes and malacostracans are united at a deeper phylogenetic level, and the free-living lecithotrophic nauplius represents a synapomorphic stage of Remipedia + Malacostraca.

(3) Alternatively, free-living lecithotrophic nauplii of Remipedia and some Malacostraca may have evolved independently.

Both scenarios 1 and 2 argue for a close relationship of Remipedia and Malacostraca. Moreover, the features shared between free-living lecithotrophic nauplii of both taxa seem to coincide with complex aspects of brain morphology (Fanenbruck et al. 2004).

Some features, nevertheless, would appear to argue for a primitive position for remipedes, viz., the uniramous first antennae in the early larva, the homonomously segmented trunk, and a mandible with well-developed palps. However, although the mandibles of these larvae remain robustly biramous until late in development, the postmandibular mouthparts are uniramous from their first appearance as Anlagen and remain so into the adult form. Of added interest is the fact that the maxilliped of these larvae emerges as a mouthpart at its earliest appearance. There is no indication that it was derived from a trunk appendage, whereas in malacostracans, maxillipeds are obviously modified thoracopods.

However, several fundamental questions regarding crustacean/arthropod development still remain unresolved. For example, there are controversial views as to which naupliar features define the Crustacea $s$. lat. (Walossek and Müller 1990; Dahms 2000, 2006; Scholtz 2000) or whether an orthonauplius (with three pairs of cephalic appendages) might represent a phylotypic stage in crustacean development (Dahms 2000, 2006). To answer these questions we need to determine whether the Crustacea s. str. are indeed mono-phyletic (or not). Most obviously, larval development should be a crucial aspect in more comprehensive phylogenetic analyses of the Arthropoda.

Nevertheless, it seems that a group of crustaceans including remipedes, cephalocarids, most of the maxillopodans, and malacostracans is quite separate from branchiopods, mystacocarids, and branchiurans (Schram and Koenemann 2004a).

So once again, as in the days of Thompson, the discovery of larvae serves to both answer questions about affinities of a group of crustaceans and also to raise new issues about the evolution of this fascinating group.

\section{Acknowledgments}

The March 2006 research expedition to Abaco was funded by grants from German Research Society (DFG KO 3483/1-1) (S. K.), and the Biodiversity Surveys and Inventories Program, NSF (DEB-0315903) (T. M. I.). Collection of specimens was carried out under Bahamas Department of Fisheries marine resource collection permit (T. M. I.). We thank Jan Michels for assistance with CLSM photography. Logistical assistance for field work in Abaco was provided by Nancy Albury (Friends of the Environment, Abaco). In addition to T. Iliffe and M. Hoenemann, other Abaco expedition team members included Renee Bishop, Lara Hinderstein, Brett Gonzalez, Ute Wollermann, Brian Kakuk, Gregg Stanton, and Tamara Thomsen.

\section{REFERENCES}

Boxshall, G. A., Ströberg, J.-O., Dahl, E. (eds.). 1992. The Crustacea: origin and evolution. Acta Zool. 73: 271-392.

Dahms, H.-U. 2000. Phylogenetic implications of the Crustacean nauplius. Hydrobiologia 417: 91-99.

Dahms, H.-U. 2006. Key for the identification of crustacean nauplii. Org. Divers. Evol. 6: 47-56.

Emerson, M. J., and Schram, F. R. 1997. Theories, patterns, and reality: game plan for arthropod phylogeny. In R. A. Fortey and R. H. Thomas (eds.). Arthropod Relationships. Chapman \& Hall, London, pp. 67-86.

Fanenbruck, M., Harzsch, S., and Wägele, J. W. 2004. The brain of the Remipedia (Crustacea) and an alternative hypothesis on their phylogenetic relationships. Proc. Natl. Acad. Sci. USA 101: 3868-3873.

Koenemann, S., Schram, F. R., and Iliffe, T. M. 2006. Trunk segmentation patterns in Remipedia. Crustaceana 79: 607-631.

McKenzie, K. G. 1991. Crustacean evolutionary events: sequences and consequences. Mem. Queensland Mus. 31. 19-38. 
Moura, G., and Christoffersen, M. L. 1996. The system of the mandibulate arthropods: tracheata and Remipedia as sister groups, "Crustacea" nonmonophyletic. J. Comp. Biol. 1: 95-113.

Scholtz, G. 2000. Evolution of the nauplius stage in malacostracan crustaceans. J. Systems Evol. Res. 38: 175-187.

Schram, F. R. 1983. Remipedia and crustacean phylogeny. In F. R. Schram (ed.). Crustacean phylogeny, Crustacean Issues 1. A. A. Balkema Press, Rotterdam, pp. 23-28.

Schram, F. R., and Hof, C. H. J. 1998. Fossil taxa and the relationships of major crustacean groups. In G. Edgecombe (ed.). Arthropod Fossils and Phylogeny. Columbia University Press, New York, pp. 233-302.

Schram, F. R., and Koenemann, S. 2004a. Developmental genetics and arthropod evolution: on body regions of Crustacea. In G. Scholtz (ed.) Evolutionary Developmental Biology of Crustacea, Crustacean Issues 15. A. A. Balkema Press, Rotterdam, pp. 75-92.

Schram, F. R., and Koenemann, S. 2004b. Are crustaceans monophyletic? In J. Cracraft and M. J. Donaghue (eds.). Assembling the Tree of Life. Oxford University Press, Oxford, pp. 319-329.

Schram, F. R., and Lewis, C. A. 1989. Functional morphology of feeding in Nectiopoda. In B. E. Felgenhauer, L. Watling, and A. B. Thistle (eds.)
Functional Morphology of Feeding and Grooming in Crustacea, Crustacean Issues. Vol. 6. A. A. Balkema Press, Rotterdam, pp. 115-122.

Schram, F. R., Yager, J., and Emerson, M. J. 1986. Remipedia. Part 1. Systematics. Mem. S.D. Soc. Nat. Hist. 15: 1-60.

Snodgrass, R. E. 1952. A Textbook of Arthropod Anatomy. Comstock Publishing Associates, Ithaca.

Thompson, J. V. 1830. On the cirripedes or barnacles. Zool Res., Vol. I, Pt. 1, Mem. IV 69-88.

van der Ham, J. L., and Felgenhauer, B. E. 2007. The functional morphology of the putative injecting apparatus of Speleonectes tanumekes (Remipedia). J. Crust. Biol. 27: 1-9.

Walossek, D., and Müller, K. J. 1990. Upper Cambrian stem-lineage crustaceans and their bearing upon the monophyletic origin of Crustacea and the position of Agnostus. Lethaia 23: 409-427.

Wills, M. 1997. A phylogeny of recent and fossil Crustacea derived from morphological characters. In R. A. Fortey and R. H. Thomas (eds.). Arthropod Relationships. Chapman \& Hall, London, pp. 189-209.

Yager, J. 1981. Remipedia, a new class of Crustacea from a marine cave in the Bahamas. J. Crust. Biol. 1: 328-333. 